\title{
Typologie Des Gîtes Larvaires Et Résistance Des Vecteurs Du Paludisme A La Deltaméthrine Dans les Milieux Urbain Et Rural Du Département De l'Atlantique Au Sud Du Bénin: Données Préliminaires
}

\author{
Innocent Djègbè, PhD \\ Université Nationale des Sciences, Technologie, Ingénierie et \\ Mathématiques d'Abomey, Ecole Normale Supérieure de Natitingou, \\ Plateforme Agro-Eco-Health, IITA/Bénin
}

Filémon Toponon, PhD

Programme National de Lutte contre le Paludisme (PNLP),

Ministère de la Santé, Bénin

Adam Gbankoto, PhD

Laboratoire de Physiologie et Pharmacologie Expérimentale, Faculté des Sciences et Techniques, Université d'Abomey-Calavi, Bénin

Geneviève Tchigossou, PhD

Plaforme Agro-Eco-Health, International Institute of Tropical Agriculture (IITA), Benin

Donald Djossou-Hessou, Msc

Camille Dossou, Msc

Université Nationale des Sciences, Technologie, Ingénierie et Mathématiques d'Abomey, Ecole Normale Supérieure de Natitingou, Plateforme Agro-Eco-Health, IITA/Bénin

Akadiri Yessoufou, PhD

Faculté des Science et Techniques; Université d'Abomey Calavi, Benin

Martin Akogbéto, PhD

Centre de Recherche Entomologique de Cotonou (CREC), Bénin

Luc Djogbénou, PhD

Institut Régional de Santé Publique, Université d'Abomey Calavi, Bénin

Rousseau Djouaka, PhD

Plaforme Agro-Eco-Health,

International Institute of Tropical Agriculture (IITA), Benin

Doi:10.19044/esj.2019.v15n33p171 URL:http://dx.doi.org/10.19044/esj.2019.v15n33p171 


\section{Résumé}

La lutte antilarvaire récemment recommandée par l'OMS, requiert une connaissance approfondie de la distribution et de la typologie des gîtes larvaires des vecteurs du paludisme. L’objectif de cette étude est d'identifier les différents gîtes larvaires des anophèles et leur mécanisme de résistance à la deltaméthrine. Des prospections larvaires ont été effectuées en 2017 durant les saisons pluvieuses et sèches dans trois communes au sud du Bénin. Les moustiques issus de l'émergence des larves ont été soumis à la deltaméthrine et au bendiocarb selon le protocole de l'OMS. L'identification moléculaire des anophèles et le génotypage de la mutation $\mathrm{Kdr}$ ont été réalisés par PCR et l'expression des oxydases, des estérases $\alpha$ et $\beta$, et des GST ont été mesurées. Les prospections larvaires ont permis de répertorier 37 gîtes larvaires regroupés en 13 types. La majorité des gîtes étaient anthropiques. La densité larvaire variait d'un type de gîtes à l'autre. An. coluzzii et An. gambiae étaient les deux vecteurs du paludisme vivant en sympatrie dans les trois sites d'étude. Ils sont fortement résistants à la deltaméthrine avec la présence de la mutation $\mathrm{kdr}$ à des fréquences très élevées et une augmentation des activités des estérases dans les populations d'anophèles collectés à Zè et des GST à Abomey-Calavi et Allada. La prolifération des vecteurs du paludisme serait imputable à l'insalubrité de l'environnement immédiat et aux activités anthropiques qui créent et assurent le maintien des gîtes larvaires. Ces données pourraient servir au renforcement des stratégies de lutte contre le paludisme déjà en cours.

Mots-clés : Anophèles, Gîte larvaire, Résistance aux insecticides, Paludisme, Bénin. 


\title{
Typology of Larval habitats and Resistance of Malaria Vectors to Deltamethrin in Urban and Rural Areas of the Atlantic Department in Southern Benin: Baseline Data
}

\author{
Innocent Djègbè, PhD \\ Université Nationale des Sciences, Technologie, Ingénierie et \\ Mathématiques d'Abomey, Ecole Normale Supérieure de Natitingou, \\ Plateforme Agro-Eco-Health, IITA/Bénin
}

Filémon Toponon, PhD

Programme National de Lutte contre le Paludisme (PNLP),

Ministère de la Santé, Bénin

Adam Gbankoto, PhD

Laboratoire de Physiologie et Pharmacologie Expérimentale, Faculté des Sciences et Techniques, Université d'Abomey-Calavi, Bénin

Geneviève Tchigossou, PhD

Plaforme Agro-Eco-Health, International Institute of Tropical Agriculture (IITA), Benin

Donald Djossou-Hessou, Msc

Camille Dossou, Msc

Université Nationale des Sciences, Technologie, Ingénierie et Mathématiques d'Abomey, Ecole Normale Supérieure de Natitingou, Plateforme Agro-Eco-Health, IITA/Bénin

Akadiri Yessoufou, PhD

Faculté des Science et Techniques; Université d'Abomey Calavi, Benin

Martin Akogbéto, PhD

Centre de Recherche Entomologique de Cotonou (CREC), Bénin

Luc Djogbénou, PhD

Institut Régional de Santé Publique, Université d'Abomey Calavi, Bénin

Rousseau Djouaka, PhD

Plaforme Agro-Eco-Health,

International Institute of Tropical Agriculture (IITA), Benin

Abstract

Anopheles larval control, recently recommended by WHO, requires a deep knowledge of the distribution and typology of larval breeding sites. The 
objective of this study is to identify the different larval habitats colonized by Anopheles and their insecticide resistance mechanism. Larval surveys were carried out in three Districts in south of Benin in 2017, during the rainy and dry seasons. Mosquitoes breeding sites have been characterized and mapped. Mosquitoes from the emergence of larvae were tested to deltamethrin and bendiocarb according to the WHO protocol. The molecular identification of anopheles and the genotyping of the kdr mutation were performed by PCR and the expression of oxidases, esterases, and GSTs was measured. Larval surveys have identified 37 breeding sites categorized into 13 types. Most of the larval habitats were anthropogenics. An. coluzzii and An. gambiae were the two malaria vectors found in sympatric in the three study sites. These two vectors were highly resistant to deltamethrin with the presence of the kdr L1014F mutation at very high frequencies and an increase in esterase activities in anopheline populations collected in Zè and GST in Abomey-Calavi and Allada. The proliferation of malaria vectors is attributable to the unhealthy environment and human activities that create and maintain mosquito breeding. This study highlighted diversity in the type of breeding site of An. gambiae s.s in the Atlantic Department, suggesting the adaptation of this species in its environment. These results could be used to develop an antilarval control strategy in Abomey-Calavi, Zè and in Allada.

Keywords: Anopheles, Breeding site, Insecticide resistance, Malaria, Benin

\section{Introduction}

Les moustiques sont responsables de la transmission d'agents pathogènes qu'ils peuvent inoculer à l'homme pendant leur repas sanguin. De ce fait, ils représentent un risque pour la santé humaine. Parmi ces moustiques on trouve le genre des Anophèles qui sont responsables de la transmission de Plasmodium, agent pathogène du paludisme en Afrique. En effet, sur plus de 3000 espèces d'anophèles enregistrées dans le monde, environ 60 transmettent le paludisme qui constitue un problème majeure de santé publique en Afrique (Mouchet et al., 2004).

En Afrique, les principaux vecteurs du paludisme sont An. gambiae s.l, An. funestus, An. nili et An. moucheti. Ces anophèles appartiennent tous à des complexes d'espèces et peuvent avoir des capacités vectorielles et des comportements très variables (Fontenille et al., 2005). Ils se reproduisent dans des points d'eau dont les caractéristiques physiques et chimiques classiques ont été décrits. Par exemple, Anopheles gambiae s.l, vecteur majeur du paludisme, se développe selon plusieurs auteurs dans des points d'eau ensoleillés, claires et propres et quant-à An. funestus s.l, il est connu inféodé aux gîtes ombragés par des végétaux aquatiques (Mouchet et al., 2004). Outre ces gîtes classiques, les hommes créent de plus en plus dans leur 
environnement immédiat, d'autres points d'eau soit par leurs habitudes de conservation d'eau dans des jarres ou par des activités économiques.

Au Bénin, malgré les efforts nationaux et internationaux, le paludisme demeure un problème majeur de santé publique avec une augmentation de l'incidence palustre et de la létalité qui a été multipliée par deux entre 2010 et 2017 (OMS, 2018). Actuellement, les deux principales méthodes de lutte antivectorielle préconisées par le Programme National de Lutte contre le Paludisme (PNLP) sont basées sur l'utilisation des insecticides. Elles ciblent les moustiques adultes à travers des pulvérisations intra-domiciliaires d'insecticides à effet rémanent (PID) et l'utilisation des moustiquaires imprégnées à longue durée d'action (MILD) ((Akogbéto et al., 2010; Ossè et al., 2012 Rowland et al., 2013; Djouaka et al., 2016; Akoton et al., 2018). Cependant, le développement de la résistance des anophèles aux insecticides et l'existence d'une transmission résiduelle lorsque les gens ne sont pas protégés par les PID ou les MILD, soulignent l'intérêt de développer des méthodes de lutte complémentaires (Djègbè et al., 2017; Djouaka et al., 2016). Parmi celles-ci, figure la lutte anti-larvaire qui a été récemment recommandée par l'OMS (OMS, 2019). Toutefois, une campagne de lutte antilarvaire passe d'abord par la connaissance approfondie de la distribution et de la typologie des gîtes colonisés par les anophèles. Si de nombreuses études ont été réalisées en Afrique sur la bio-écologie des vecteurs (Etang et al., 2016; Kudom, 2015; Saliha et al., 2018), une seule étude décrivant les habitats larvaires des anophèles dans un village lacustre a été réalisée au Bénin (Akogbéto, 1995). Donc, Il est urgent de disposer des informations actualisées sur la bio-écologie des larves de moustique afin de connaître les caractéristiques des biotopes et leur différentiation dans l'espace et de dégager les conditions favorables pour le développement des moustiques (Berchi et al., 2012; El Ouali Lalami et al., 2011; Mesari, 1997). C'est dans ce cadre que la présente étude a été menée et elle a pour objectifs: d'identifier les différents gîtes colonisés par les anophèles vecteurs du paludisme et de déterminer leur sensibilité à la deltaméthrine, insecticide utilisé dans l'imprégnation des moustiquaires de la population béninoise. Ainsi, les résultats de cette étude peuvent être servir dans l'utilisation potentielle des méthodes de lutte complémentaires basées sur le contrôle des stades larvaires dans le département de l'Atlantique au Bénin.

\section{Matériels et Méthodes}

\section{- Sites d'études}

L'étude a été réalisée dans trois communes urbaines et rurales du département de 1'Atlantique au Sud Bénin à savoir Abomey-Calavi (commune urbaine, $6^{\circ} 26^{\prime} 55^{\prime \prime}, 2^{\circ} 21^{\prime} 20^{\prime \prime}$ ), Zè (commune rurale, $6^{\circ} 47^{\prime} 00^{\prime \prime}, 2^{\circ} 18^{\prime} 00^{\prime \prime}$ ) et Allada (commune rurale, 6 $6^{\circ} 39^{\prime} 54^{\prime \prime}, 2^{\circ} 09^{\prime} 08^{\prime \prime}$ ) (Figure 1). Ces communes présentent des conditions favorables à la prolifération des moustiques. Le 
département de l'Atlantique est caractérisé par deux saisons sèches (une grande saison sèche allant de Décembre à Mars et une petite saison sèche d'Août à Septembre) et deux saisons pluvieuses (une grande saison pluvieuse allant d'Avril à Juillet et une petite saison pluvieuse d'Octobre à Novembre). La température moyenne annuelle est de $27,4^{\circ} \mathrm{C}$ (Boko, 1992) .

Abomey-Calavi : Cette commune urbaine, a un relief peu accidenté. Les principaux traits caractéristiques sont une bande sablonneuse avec des cordons littoraux, un plateau de terre de barre avec des dépressions et marécages. Le climat est de type sub-équatorial. La moyenne des précipitations annuelles est de $1179 \mathrm{~mm}$. Le réseau hydrographique est constitué essentiellement de deux plans d'eau que sont le lac Nokoué et la lagune côtière. Par ailleurs, la commune dispose d'une façade maritime juxtaposée à la lagune côtière, des marais, des ruisseaux et des marécages (Biaou, 2006).

Zè : le relief de cette commune rurale est un élément du vaste plateau d'Allada d'une altitude moyenne de $100 \mathrm{~m}$ qui s'incline légèrement vers la côte et surplombe au nord la dépression de la Lama. Il est composé de quelques petites dépressions constituées de bas-fonds. Son climat est de type béninien avec une pluviométrie moyenne annuelle de $1217 \mathrm{~mm}$. Le réseau hydrographique peu dense est alimenté par les affluents du fleuve Ouémé tel que la Sô et parsemé de plusieurs bas-fonds. Le couvert végétal est composé de forêts claires, de formations boisées et de formations arborées et arbustives (Fahala, 2006).

Allada : la commune rurale d'Allada est située dans la zone du plateau de terre de barre qui descend vers les vallées des fleuves Ouémé, et Couffo, et la dépression de la Lama. Le sol d'Allada est essentiellement caractérisé par la terre de barre et une dépression marécageuse. Le réseau hydrographique est composé du lac Ahémé et une rivière du Couffo. Allada disposait d'une forêt dense qui a disparu sous l'effet de la pression démographique et des défrichements abusifs, laissant place à une savane arborée (Nanagbé, 2006). 


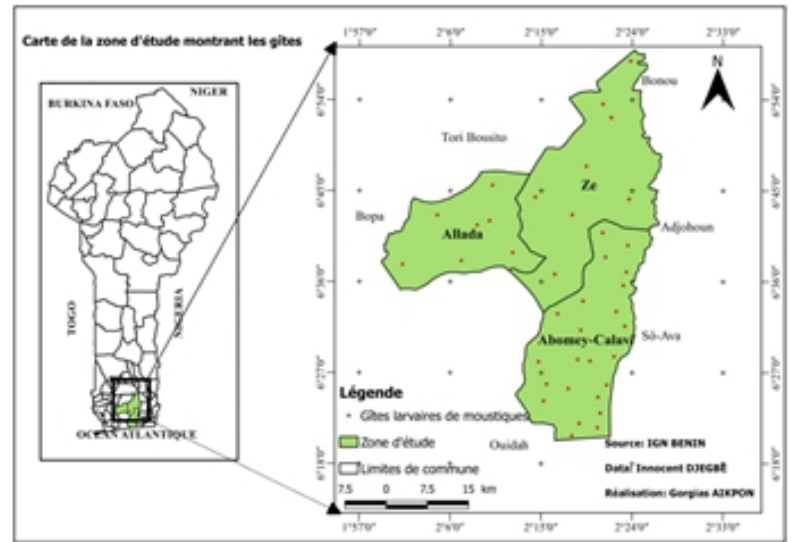

Figure 1 : Carte montrant les trois sites d'étude avec la distribution des gîtes larvaires de moustiques

- Prospection, typage et cartographie des habitats larvaires de moustiques

La prospection des gîtes larvaires s'est déroulée en deux périodes : en Juin 2017 (la grande saison des pluies) et en Décembre 2017 (la grande saison sèche). Elle a consisté à repérer les gîtes larvaires potentiels. Les collections d'eau dans lesquelles les larves (tous stades confondus) de moustiques ont été observées sont considérées comme des gîtes larvaires. Chaque gîte identifié a été typé en fonction du contenant et géo-référencé à l'aide d'un GPS de marque GARMIN@ et les données ont été importées dans le logiciel de cartographie Arc GIS version 10.1 afin de les positionner sur un modèle numérique de terrain.

\section{- Collection des larves et détermination de la densité larvaire}

Les stades immatures des moustiques ont été collectés par la méthode de dipping (Silver \& Service, 2007) en utilisant des louches à manche de volume $200 \mathrm{ml}$, dans les différents points d'eau stagnants trouvés dans les trois localités. La densité larvaire a été déterminée en dénombrant tous les stades larvaires après une série de 10 prélèvements et en standardisant le volume à 1 litre. Ces formes immatures ont été triées, rincées avec de l'eau propre et remises dans de gobelets en plastique étiquetés puis transportées à l'insectarium de l'Institut International d'Agriculture Tropicale (IITA-Bénin) où elles ont été élevées jusqu'à l'émergence des adultes. L'élevage s'est déroulé dans des conditions standard de température $\left(25\right.$ à $\left.28^{\circ} \mathrm{C}\right)$ et d'humidité (70 à $80 \%$ ) à l'insectarium de l'IITA-Bénin. Les formes adultes obtenues ont été utilisées pour les tests de sensibilités selon le protocole de l'Organisation Mondiale de la Santé (OMS, 2017).

\section{- Tests de sensibilité aux insecticides}

Les tests de sensibilité ont été réalisés sur la souche Kisumu d'An. gambiae, souche sensible de référence et maintenue à une température de $25^{\circ} \mathrm{C}$ 
et une humidité relative de $80 \%$ à l'insectarium de la Plateforme Agro-EcoHealth de l'Institut International d'Agriculture Tropicale (IITA-Bénin) et sur les femelles à jeun et âgées de 3 à 5 jours, issues des populations naturelles d'An. gambiae s.l. provenant respectivement des trois communes : d'AbomeyCalavi, Zè et Allada. Les insecticides utilisés étaient la deltaméthrine $(0,05 \%)$ de la classe des pyréthrinoïdes et le bendiocarb $(0,1 \%)$ de la classe des carbamates. Ces deux insecticides sont utilisés au Bénin respectivement pour les moustiquaires imprégnées et les pulvérisations intra-domiciliaires. Les tests consistaient à exposer pendant une heure les moustiques aux doses diagnostiques d'insecticide sur papier imprégné et à déterminer leur taux de mortalité après 24 heures d'observation. Des lots de 20-25 anophèles par tube étaient exposés aux papiers imprégnés d'insecticide pendant une heure, la durée au cours de laquelle les moustiques assommés ont été dénombrés à des intervalles de temps réguliers de 5 minutes. Durant le temps d'observation qui a duré $24 \mathrm{~h}$, les moustiques ont été nourris de solution de saccharose $10 \%$. Des tests utilisant des papiers non traités ont été considérés comme témoins. $\mathrm{Au}$ terme des tests de sensibilités aux insecticides, tous les adultes d'Anopheles gambiae s.l testés (morts et survivants) provenant de chaque site, ont été conservés dans des tubes Eppendorf de 1,5 ml contenant du silicagel. En effet, ces anophèles ont été conditionnés de façon séparée par lot, les morts formaient un lot et les survivants un autre. Ces deux lots de spécimens ont servi pour l'identification moléculaire des espèces de moustiques et la détermination des mutations Kdr L1014F.

- Identification des moustiques et détection du mécanisme de résistance kdr L1014F)

L'identification des adultes issus de l'élevage des larves collectées sur le terrain, a été réalisée sur la base des critères morphologiques définis par les clés d'identification (Gillies \& De Meillon, 1968). Les espèces du complexe An. gambiae s.l ont été identifiées par la technique de «Polymerase Chain Reaction-Restriction Fragment Length Polymorphism» (PCR-RFLP) (Fanello et al., 2003). La détection du mécanisme de résistance kdr (1014F) des vecteurs aux insecticides a été effectuée par la technique de PCR Taqman (Bass et al., 2008).

- Mesure de l'activité des enzymes de détoxification des insecticides

Des tests biochimiques ont été réalisés pour quantifier le niveau des enzymes de détoxification notamment les oxydases, l'activité non spécifique de l'estérase et de la GST chez les individus femelles âgés de 3 à 5 jours non exposés aux insecticides. Un total de 150 moustiques, à raison de 50 moustiques pour chacun des sites a été analysé. Ces échantillons ont été testés sur plaque de micro-titrage, par spectrophotométrie (Hemingway, 2004). 


\section{- Analyse statistique des données}

Les statuts de résistance et de sensibilité aux insecticides des moustiques testés ont été évalués selon les critères de l'OMS (OMS, 2017). La fréquence de la mutation kdr L1014F a été comparée entre An. coluzzii et An. gambiae, par le test de khi2 à l'aide du logiciel Statistica. Les données biochimiques (activité enzymatique par mg de protéine) ont été comparées entre la souche de référence (Kisumu) et les moustiques de terrain par le test non paramétrique de Kruskal-Wallis.

\section{Résultats}

\section{- Typologie des gîtes}

Au total, 37 gîtes larvaires ont été identifiés dans les trois localités prospectées au cours des deux enquêtes soit 23 à Abomey-Calavi, 8 à Zè et 7 à Allada. Ces gîtes ont été regroupés en 13 catégories présentées en figure 2 . Il s'agit des mares $(29,73 \%)$, des caniveaux $(2,70 \%)$, des pneus usés $(10,81 \%)$, des chambres à air $(5,41 \%)$, des pots en argiles $(8,11 \%)$, des conteneurs métalliques $(2,70 \%)$, des marécages $(2,70 \%)$, des pirogues abandonnées $(8,11 \%)$, écorce d'arbre $(2,270 \%)$, des bidons usés $(13,51 \%)$, épave d'engin de dragage $(2,70 \%)$, des adductions d'eau défectueuses $(2,70 \%)$ et des bassins de stockage d'eau $(8,11 \%)$. Les mares $(29,73 \%)$ ont été les gîtes les plus fréquents alors que les caniveaux, conteneurs métalliques, marécage, écorce d'arbre, adduction d'eau défectueuse et épave d'engin de dragage ont été les moins fréquents $(2,70 \%)$ sur l'ensemble des sites d'études (Tableau 1).

Tableau 1 : Typologie des gîtes larvaires et leurs fréquences d'apparition dans les sites d'étude

\begin{tabular}{|c|c|c|c|c|c|c|c|c|}
\hline \multirow[b]{2}{*}{ Type de gîtes } & \multicolumn{2}{|c|}{$\begin{array}{l}\text { Abomey- } \\
\text { Calavi }\end{array}$} & \multicolumn{2}{|r|}{ Zè } & \multicolumn{2}{|c|}{ Allada } & \multicolumn{2}{|c|}{ Total } \\
\hline & $\mathrm{N}$ & $\%$ & $\mathrm{n}$ & $\%$ & $\mathrm{n}$ & $\%$ & $\mathrm{n}$ & $\%$ \\
\hline Mares & 6 & 26,09 & 2 & 25,00 & 3 & 42,86 & 11 & 29,73 \\
\hline Caniveaux & 1 & 4,35 & 0 & 0,00 & 0 & 0,00 & 1 & 2,70 \\
\hline Pneus usés & 2 & 8,70 & 0 & 0,00 & 0 & 0,00 & 2 & 5,41 \\
\hline Pot en argile & 0 & 0,00 & 2 & 25,00 & 1 & 14,29 & 3 & 8,11 \\
\hline Chambre à air usé & 3 & 13,04 & 0 & 0,00 & 1 & 14,29 & 4 & 10,81 \\
\hline Conteneur métallique & 1 & 4,35 & 0 & 0,00 & 0 & 0,00 & 1 & 2,70 \\
\hline Marécages & 1 & 4,35 & 0 & 0,00 & 0 & 0,00 & 1 & 2,70 \\
\hline Ecorce d'arbre & 0 & 0,00 & 1 & 12,50 & 0 & 0,00 & 1 & 2,70 \\
\hline Pirogue abandonnée & 2 & 8,70 & 1 & 12,50 & 0 & 0,00 & 3 & 8,11 \\
\hline $\begin{array}{l}\text { Bidon abandonné } \\
\text { Adduction d'eau }\end{array}$ & 3 & 13,04 & 1 & 12,50 & 1 & 14,29 & 5 & 13,51 \\
\hline défectueuse & 0 & 0,00 & 1 & 12,50 & 0 & 0,00 & 1 & 2,70 \\
\hline $\begin{array}{l}\text { Bassin de stockage d'eau } \\
\text { Epaves d'engin de }\end{array}$ & 3 & 13,04 & 0 & 0,00 & 1 & 14,29 & 3 & 8,11 \\
\hline dragage & 1 & 4,35 & 0 & 0,00 & 0 & 0,00 & 1 & 2,70 \\
\hline Total & 23 & 100,00 & 8 & 100,00 & 7 & 100,00 & 37 & 100,00 \\
\hline
\end{tabular}




\section{- Diversité et moyenne des densités larvaires de culicidés collectés} sur les sites d'étude

Les gîtes rencontrés à Abomey-Calavi étaient de dix types : mares, caniveaux, pneus usés, chambre à air usée, marécage, conteneur métallique, pirogue abandonnée, épave d'engin de dragage, les bidons abandonnés et les bassins de stockage d'eau. Les mares sont les gîtes les plus rencontrés avec une densité larvaire moyenne de 10 larves/litre. La plus forte densité larvaire était de 25 larves/litre dans un gîte constitué d'épave d'engin de dragage alors que la plus faible densité larvaire était de 1 larve/litre dans un gîte constitué de pot en argile.

Les gîtes rencontrés à Zè sont de six types : mares, pot en argile, bidon abandonné, pirogue abandonnée, adduction d'eau défectueuse et écorce d'arbre. Les mares sont aussi les plus fréquents avec une densité larvaire moyenne de 9 larves/litre. Les gîtes en pot d'argile avaient une densité larvaire moyenne de 3 larves/litre.

Les gîtes rencontrés à Allada sont de cinq types constitués de mares, pot en argile, chambre à air usé, bidon abandonné et bassin de stockage d'eau. La plus forte densité larvaire a été obtenue dans les mares avec 15 larves/litre (Tableau 2). Les gîtes les moins productifs sont les chambres à air usés avec une densité moyenne de 3 larves/litres.

Les genres de moustiques collectés dans ces différents types de gîtes sont Anopheles, Culex et Aedes (Tableau 2). La richesse spécifique variait d'un type de gîte à l'autre. Parmi les 13 types de gîtes répertoriés, An. gambiae s.l a été retrouvé seul dans les gîtes constitués de marécages, d'épave d'engin de dragage, d'adduction d'eau défectueuse et de bassin de stockage. Les gîtes dans lesquels An. gambiae s.l a été retrouvé en sympatrie avec Culex ou Aedes sont les caniveaux, les conteneurs métalliques, les pirogues abandonnées et les mares (Tableau 2).

Tableau 2 : Diversité et moyennes des densités larvaires de culicidés selon le type de gîte dans les sites d'études

\begin{tabular}{cccc}
\hline $\begin{array}{c}\text { Sites } \\
\text { d'étude }\end{array}$ & Types de gîtes & $\begin{array}{c}\text { Densité } \\
\text { larvaire } \\
\text { moyenne } \\
\text { (larve/litre) }\end{array}$ & $\begin{array}{c}\text { Diversités } \\
\text { Culicidiennes }\end{array}$ \\
\hline $\begin{array}{c}\text { Abomey- } \\
\text { Calavi }\end{array}$ & Mares & 10 & Anopheles, culex \\
& Caniveaux & 3 & culex, Anopheles \\
& Pneus usés & 10 & Aedes, Culex \\
& Chambre à air usé & 10 & Aedes, Culex \\
& Conteneur métallique & 4 & Anopheles, Culex \\
& Marécages & 15 & Anopheles \\
& Pirogue abandonnée & 5 & Aedes, Culex, Anopheles \\
& Bidon abandonné & 6 & Aedes \\
Récipient en terre cuite & 1 & culex,
\end{tabular}


Carcasse d'engin de dragage

Zè

Allada

Mares
Pot en argile
Chambre à air usé
Bidon abandonné
Bassin de stockage d'eau

\section{9}

3

15

6

4

5

15

4

3

8
Anopheles

Anopheles

Aedes

Culex

Anopheles

Aedes

Anopheles

Anopheles, Culex

Aedes, Culex

Aedes, Culex

Aedes

Anopheles

\section{- Profil de résistance aux insecticides chez les anophèles collectés}

Les tests de sensibilité ont permis d'enregistrer des taux de mortalité de $100 \%$ pour la souche de référence Kisumu après $24 \mathrm{~h}$ avec la deltaméthrine et le bendiocarb. Par contre pour les populations de terrain, après exposition à la deltaméthrine, ces taux de mortalité étaient respectivement de 52\% chez les anophèles collectés à Abomey-Calavi, $82 \%$ à Allada et $86 \%$ à Zè. Ces taux de mortalité observés indiquent une résistance à la deltaméthrine chez les anophèles collectés dans les différents sites d'étude avec une résistance plus forte dans les populations d'Abomey-Calavi. Ces populations d'anophèles demeurent sensibles au bendiocarb avec un taux de mortalité de 100\% (voir figure 3).

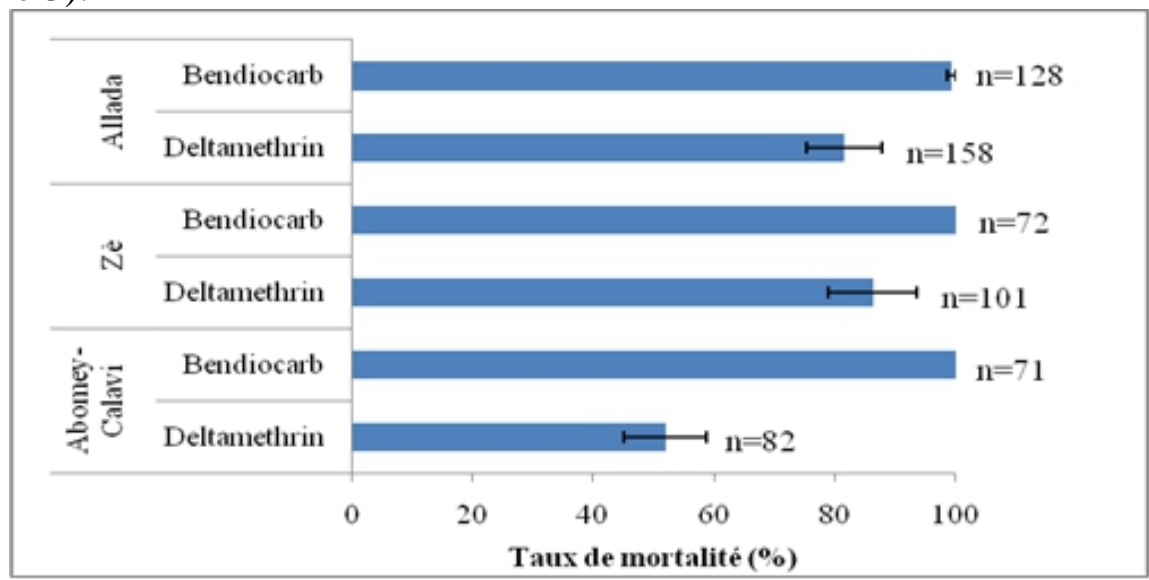

Figure 2 : Taux de mortalité $(\%)$ des anophèles après 24 heures 
- Composition des espèces d'anophèles collectées et mécanismes de résistance aux insecticides

Sur les trois sites d'étude, les deux espèces d'anophèle rencontrées sont An. coluzzii et An. gambiae, en sympatrie avec des proportions variables suivant les sites. An. coluzzii prédomine dans les gîtes rencontrés à AbomeyCalavi (54\% contre $46 \%$ pour An. gambiae), Zè (50\% contre $40 \%$ pour An. gambiae). Par contre à Allada, An. gambiae est l'espèce la plus prédominante (90\% contre 10\% de An. coluzzii). Deux mécanismes de résistance ont été observés chez les moustiques: la mutation kdr L1014F et la résistance enzymatique.

\section{- Mutation kdr L1014F}

Chez les deux espèces d'anophèle la fréquence de cette mutation est très élevée sur l'ensemble des sites d'étude et varie entre 0,8 et 0,96 . Les tests statistiques n'ont signalé aucune différence significative de ces fréquences entre les espèces et entre les sites de collecte des moustiques. Les moustiques morts et survivants après l'exposition à la deltaméthrine présentaient de fortes fréquences alléliques de la mutation Kdr L1014F chez An. Coluzzii et An. gambiae sur les trois sites d'étude (Tableau 3).

Tableau 3 : Fréquences alléliques de la mutation L1014F dans les populations naturelles de An. coluzzii et An. gambiae

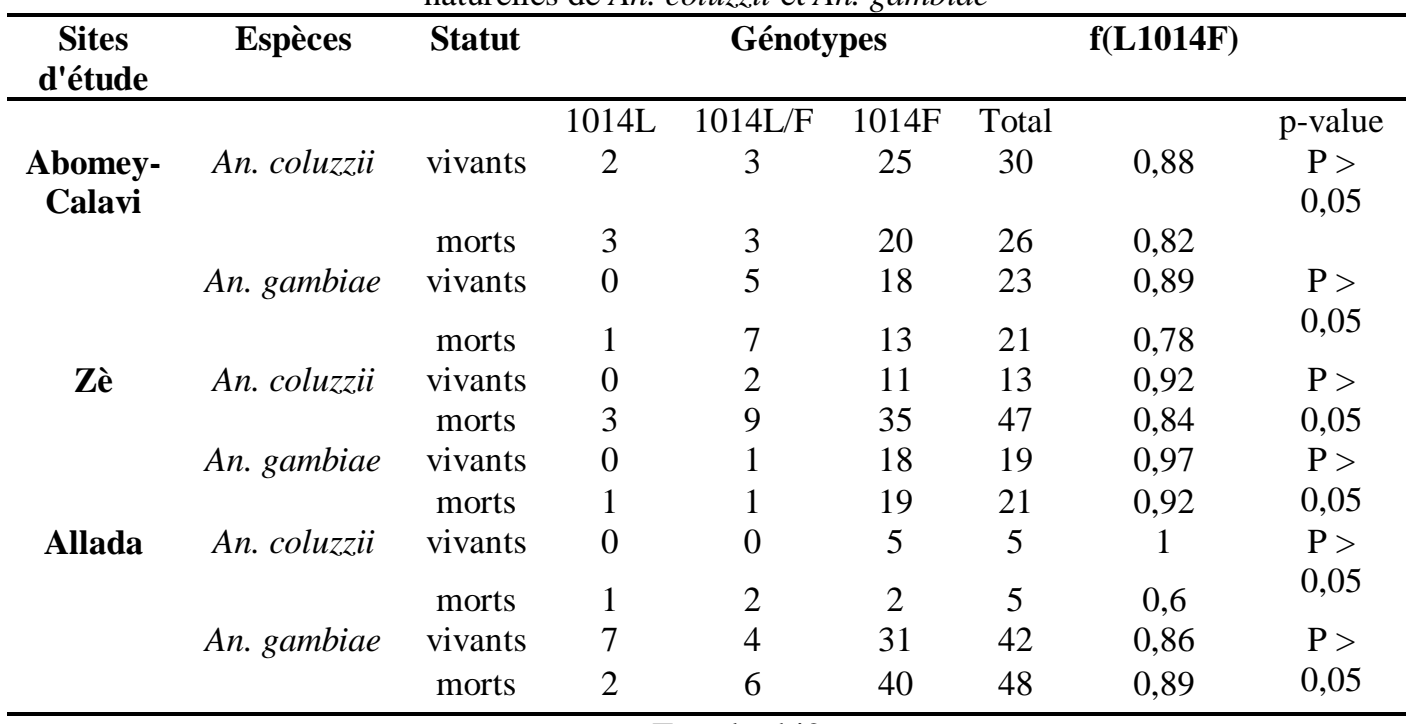

Test de chi2 


\section{- Enzymes de détoxification}

Les tests biochimiques réalisés ont montré que l'activité des estérases alpha et bêta était significativement élevée seulement dans la localité de Zè comparativement à la souche sensible de référence Kisumu $(\mathrm{p}=0,002)$. Par contre l'activité des oxydases était similaire dans toutes les localités comparativement à la souche sensible Kisumu. L'activité des GST était forte dans les localités d'Abomey-Calavi et d'Allada comparativement à la souche sensible de référence Kisumu $(\mathrm{p}=0,010)$. A Zè, cette activité était similaire à celle de Kisumu ( $\mathrm{p}=0,0562)$ (Figure 3).
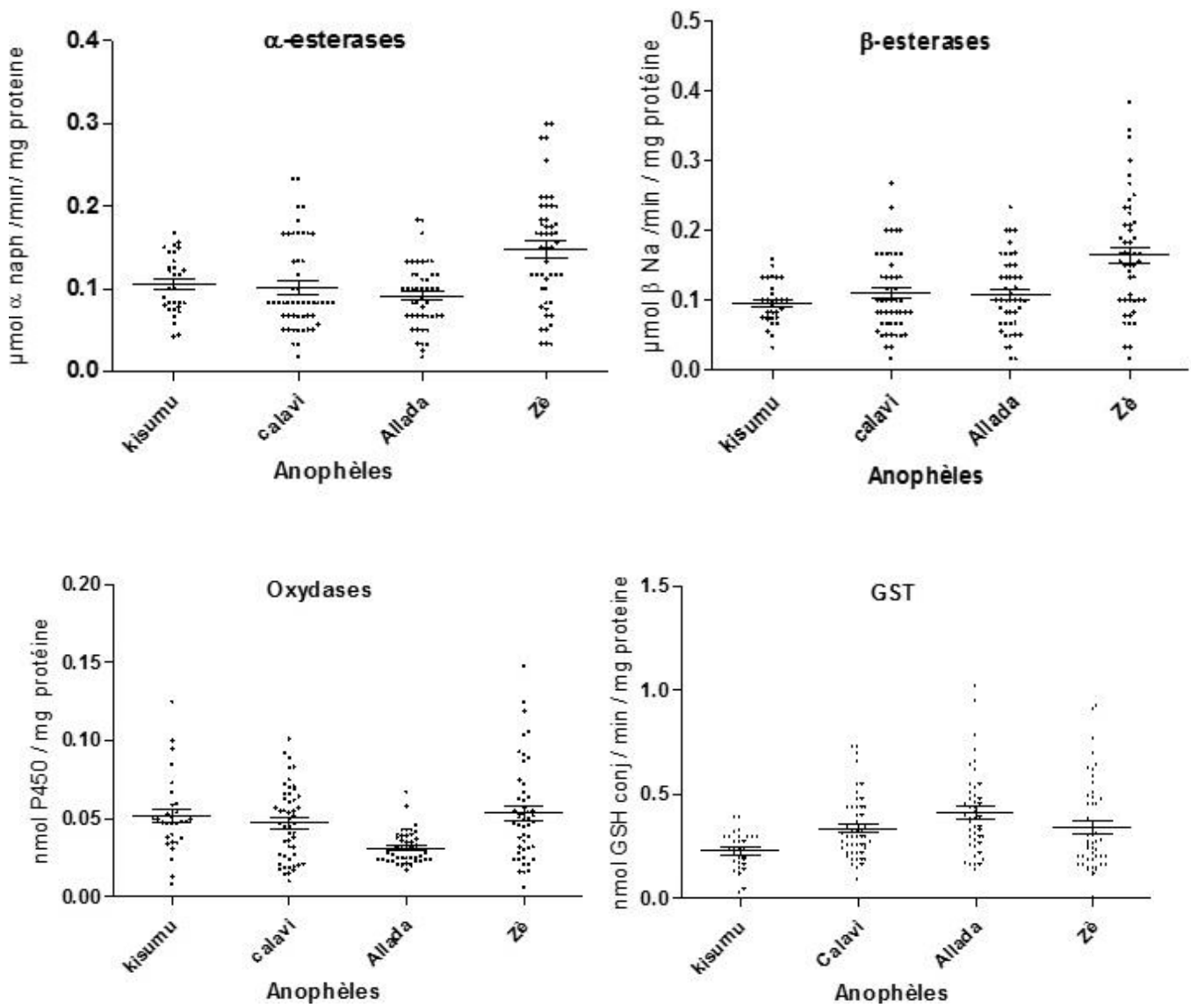

Figure 3 : Expressions des enzymes métaboliques chez les anophèles collectés sur les sites d'étude

\section{Discussion}

La présente étude, menée sur la typologie et la cartographie des habitats larvaires a permis de répertorier la diversité des gîtes de moustiques dans les trois sites d'étude du département de l'Atlantique au sud du Bénin. Ces résultats sont conformes à ceux enregistrés dans plusieurs localités rurales 
et urbaines d'Afrique (Etang et al., 2016; Koumba et al., 2018). La grande majorité des habitats larvaires rencontrés ont été des gîtes artificiels créés par l'homme à cause de ses activités économiques et industrielles. Egalement, l'urbanisation mal contrôlée, le manque des mesures d'hygiène appropriée dans nos villes et campagnes et le manque d'assainissement des alentours des habitations des villages sont à l'origine d'une telle multiplicité des gîtes de ponte de moustiques (Bouabida et al., 2012; Carnevale et al., 2009; Rodhain \& Pérez, 1985). Ces résultats rejoignent ceux obtenus par Koumba et al. (2018) dans les espaces agricoles de la zone de Mouilla au Gabon et de Saliha et al. (2018), dans la région de Bousaâda en Algérie.

Dans l'ensemble des trois communes, les moustiques pondent dans des gîtes constitués de mares, de caniveaux, de pneus usés, de pirogues abandonnées, des récipients métalliques, des épaves d'engin de dragage de sable. La majorité des gîtes (23/37) a été rencontrée dans la commune d'Abomey-calavi, une zone urbaine. Ceci est dû au fait qu'en milieux urbains, les gîtes larvaires sont accessibles et facilement repérables contrairement aux zones rurales. Ces types de gîtes larvaires de moustiques ont été couramment observés dans d'autres régions d'Afrique (Gouagna et al., 2011; Ouattara et al., 2014; Mbida et al., 2018).

Les larves d'anophèle ont été retrouvées dans les mêmes types de gîtes que les larves de Culex et d'Aedes. Il s'agit des mares, des caniveaux, des pirogues abandonnées, des conteneurs métalliques, de pneus usés. Ces observations confirment celles de Akogbéto. (1995) au sud du Bénin et Gouagna et al. (2011) dans l'Île de la Réunion. Ces différents types de gîtes présentent des densités larvaires variables d'un gîte à un autre et d'un site à un autre. La forte densité larvaire observée chez les moustiques du genre Culex à Abomey-Calavi, témoigne d'une urbanisation qui s'est accompagnée de la pollution des collections d'eau par les déchets ménagers qui sont favorables à la prolifération des larves de Culex (Hadji et al., 2013). Des observations similaires ont été effectuées par Darriet et al. (1986) et ont permis de qualifier le genre Culex de marqueur biologique de l'urbanisation au Burkina-Faso. La vie en sympatrie des anophèles et de Culex dans les mêmes gîtes confirme la capacité d'adaptation des anophèles à des gîtes pollués. Les espèces d'anophèles identifiées au cours de cette étude sont An. gambiae et An. coluzzii. Ces deux espèces ont été retrouvées en sympatrie dans les trois sites d'étude mais à des proportions variables avec une prédominance de An. coluzzii dans les sites de Abomey-Calavi et de Zè. Par contre An. coluzzii a été retrouvé en très faible proportion par rapport à An. gambiae dans la localité d'Allada. Cet état de chose serait lié au caractère rural très peu développé de la localité d'Allada par rapport à Abomey-Calavi et Zè. Ces résultats confirment ceux de Djègbè et al. (2011) et de Gnanguenon et al. (2015) au Sud du Bénin. 
Les résultats des tests de sensibilité aux insecticides traduisent une résistance de An. coluzzii et de An. gambiae à la deltaméthrine sur les trois sites d'étude. Ces résultats corroborent avec ceux des travaux de Djouaka et al. (2018), Gnanguenon et al. (2015), et Djegbe et al. (2017). L'usage massif des pyréthrinoïdes contre les ravageurs de cultures depuis les années 1970 puis leur utilisation contre les vecteurs du paludisme à travers de vastes campagnes de distribution des MILD depuis 2007 a pu contribuer à sélectionner cette résistance (Czeher et al., 2008; Djènontin et al., 2010).

Cette problématique de résistance des anophèles aux pyréthrinoïdes est particulièrement généralisée dans l'ensemble du pays et à tendance à s'aggraver d'année en année avec des répercussions réelles sur l'efficacité des MILD en termes de protection des populations vis-à-vis des moustiques vecteurs de maladies (Corbel et al., 2012).

La résistance à la deltaméthrine qui est observée chez An. gambiae et An. coluzzii serait le fait d'une insensibilité nerveuse résultant d'une modification de cible (canaux sodiques) régie par le gène $\mathrm{kdr}$ L1014F et des enzymes de détoxification (glutathion-S-transférases, Oxydases). D'ailleurs, de forte activité des glutathion-S-transférases a été observée dans les populations de moustiques d'Abomey-Calavi et d'Allada. Aussi, la présence du gène kdr observée aussi bien chez les moustiques survivants et morts après les tests de sensibilité, à des fréquences similaires suggère la présence d'autres mécanismes de résistance (résistance comportementale, résistance cubiculaire, etc.) chez ces vecteurs.

Dans cette étude, la sensibilité au bendiocarb observée chez les anophèles permet d'envisager son utilisation pour les PID dans le département de l'Atlantique puisqu'un début de résistance a été observé dans d'autres régions du pays (Aïkpon et al., 2013; Padonou et al., 2011). Toutefois, des études complémentaires permettant de faire des corrélations entre la nature des gîtes, le degré de pollution des gîtes, les paramètres physico-chimiques et microbiologiques des gîtes et la productivité des gîtes d'une part et les niveaux de résistance aux insecticides d'autre part sont à encourager. Ainsi, les informations issues de cette étude sont d'une importance capitale dans la mise en place des stratégies efficaces de lutte antivectorielle en adéquation avec les spécificités propres à chaque localité.

\section{Remerciements}

Les auteurs remercient la Banque Mondiale et le Programme National de Lutte contre le Paludisme du ministère de la santé du Bénin pour avoir financé cette étude. 


\section{Conclusion}

La présente étude a permis de catégoriser et de cartographier les différents gîtes larvaires colonisés par les vecteurs du paludisme dans trois communes urbaine (Abomey-Calavi) et rurales (Allada et Zè) du département de l'Atlantique au Sud du Bénin. La plupart de ces gîtes prospectés sont artificiels et issus des activités anthropiques. Au vu des résultats obtenus, la prolifération des moustiques vecteurs de la maladie du paludisme serait imputable à l'insalubrité de l'environnement immédiat et aux activités anthropiques qui créent et assurent le maintien des lieux de reproduction des moustiques. Cette étude met également l'accent sur la plasticité d'An. coluzzii et d'An. gambiae et sa capacité à pondre et à se développer dans des gîtes artificiels habituellement colonisés par les moustiques des genres Culex et Aedex. La résistance à la deltaméthrine des vecteurs du paludisme qui ne cesse de s'aggraver dans le pays suscite des inquiétudes quant à l'efficacité des MILD actuellement utilisées pour la lutte contre ces vecteurs. Ainsi, les données de cette étude véhiculent des informations importantes pour la conception des stratégies de lutte anti-larvaire efficaces.

\section{References:}

1. Aïkpon, R., Agossa, F., Ossè, R., Oussou, O., Aïzoun, N., Oké-Agbo, F., \& Akogbéto, M. (2013). Bendiocarb resistance in Anopheles gambiae s.l. populations from Atacora department in Benin, West Africa: A threat for malaria vector control. Parasites and Vectors, 6(1), 1-7. https://doi.org/10.1186/1756-3305-6-192

2. Akogbéto, M. (1995). Etude entomologie sur la transmission du paludisme côtier lagunaire: cas d'un village construit sur un lac d'eau saumâtre. Ann. Soc. Belge Méd Trop., 75, 219-227.

3. Akogbéto, M. C., Padonou, G. G., Gbénou, D., Irish, S., \& Yadouleton, A. (2010). Bendiocarb , a potential alternative against pyrethroid resistant Anopheles gambiae in Benin , West Africa. Malaria Journal, 9(204), 1-9. http://www.malariajournal.com/content/9/1/204

4. Akoton, R., Djègbè, G. M. T. I., Yessoufou, A., Atoyebi, M. S., Tossou, E., Zeukeng, F., Boko, P., Irving, H., Adéoti, R., Riveron, J., Wondji, C.S., Moutairou, K., Djouaka, R. (2018). Experimental huts trial of the efficacy of pyrethroids / piperonyl butoxide (PBO) net treatments for controlling multi-resistant populations of Anopheles funestus $\mathrm{s}$. $\mathrm{s}$. in Kpomè , Southern Benin, 1-18. https://doi.org/10.12688/wellcomeopenres.14589.1

5. Bass, C., Nikou, D., Blagborough, A. M., Vontas, J., Sinden, R. E., Williamson, M. S., \& Field, L. M. (2008). PCR-based detection of Plasmodium in Anopheles mosquitoes: A comparison of a new high- 
throughput assay with existing methods. Malaria Journal, 7, 1-9. https://doi.org/10.1186/1475-2875-7-177

6. Biaou, C.F 2006; Monographie de la commune d'Abomey-Calavi, Programme d'appui au démarrage des communes, Afrique Conseil, $\mathrm{P}$ 10

7. Berchi, s., Aouati, a., \& Louadi, k. (2012). Typologie des gîtes propices au développement larvaire de Culex pipiens L . 1758 ( Diptera-Culicidae ), source de nuisance à Constantine ( Algérie ). Ecologia Mediterranea, 38(2), 1-16.

8. Boko M (1992). Saisons et types de temps au Bénin : analyse objective et perceptions populaires. Espace géographique, tome 21, n4, 1992. pp. 321-332; https://doi.org/10.3406/spgeo.1992.3106

9. Bouabida, H., Djebbar, F., \& Soltani, N. (2012). Etude systématique et écologique des Moustiques ( Diptera: Culicidae ) dans la région de Tébessa (Algérie). Faunistic Entomology, 65(Figure 1), 99-103.

10. Carnevale, P., Robert, V., Manguin, S., Corbel, V., Fontenille, D., \& Garros, C. (2009). Les anophèles: biologie, transmission du Plasmodium et lutte antivectorielle (IRD, Ed.). Marseille

11. Corbel, V., Akogbeto, M., Damien, G. B., Djenontin, A., Chandre, F., Rogier, C., Moiroux, N., Chabi, J., Banganna, B., Padonou, G.G., Henry, M. (2012). Combination of malaria vector control interventions in pyrethroid resistance area in Benin: a cluster randomised controlled trial. The Lancet Infectious Diseases, 12(8), 617-626. https://doi.org/10.1016/S1473-3099(12)70081-6

12. Czeher, C., Labbo, R., Arzika, I., \& Duchemin, J. (2008). Evidence of increasing Leu-Phe knockdown resistance mutation in Anopheles gambiae from Niger following a nationwide long-lasting insecticidetreated nets implementation. Malaria Journal, 12, 1-12. https://doi.org/10.1186/1475-2875-7-189

13. Darriet, F., Carnevale, P., Rovert, V., \& White, G. B. (1986). Evaluation en phase II, dans la station expérimentale de SOUMOUSSO (Burkina-Faso) de l'efficacité sur les vecteurs du paludisme, d'un insecticide: l'OMS-3021 en aspersions intrdomiciliaires sélectives ou totales de cases Bobo et Mossi, à l'aide d'un pulvérisateur électrodynamique; Centre Muraz/OCCGE/ORSTOM, (87-8992), 31 p. multigr. (Documents Techniques OCCGE 87-8992). http://www.documentation.ird.fr/hor/fdi:24649

14. Djègbè, I., Boussari, O., Sidick, A., Martin, T., Ranson, H., Chandre, F., Akogbeto, M., Corbel, V. (2011). Dynamics of insecticide resistance in malaria vectors in Benin: First evidence of the presence 
of L1014S kdr mutation in Anopheles gambiae from West Africa. Malaria Journal, 10, 1-11. https://doi.org/10.1186/1475-2875-10-261

15. Djegbe, I., Missihoun, A. A., Djouaka, R., \& Akogbeto, M. (2017). Surveillance Entomologique: Dynamique de la population et de la résistance aux insecticides chez Anopheles gambiae s.l en milieu de riziculture irriguée au Sud Bénin . Journal of Applied Biosciences 111: 10934-10943. http://dx.doi.org/104314/jab.v111i1.10

16. Djènontin, A., Bio-Bangana, S., Moiroux, N., Henry, M. C., Bousari, O., Chabi, J., Ossè, R., Koudénoukpo. S, Corbel, V., Akogbéto, M., Chandre, F. (2010). Culicidae diversity, malaria transmission and insecticide resistance alleles in malaria vectors in Ouidah-KpomasseTori district from Benin (West Africa): A pre-intervention study. Parasites and Vectors, 3(1), 1-7. https://doi.org/10.1186/1756-3305-383

17. Djouaka, R., Riveron, J. M., Yessoufou, A., Tchigossou, G., Akoton, R., Irving, H., Djègbè, I., Moutairou, K., Adéoti, R., Tamò, M., Manyong, V., Wondji, C. S. (2016). Multiple insecticide resistance in an infected population of the malaria vector Anopheles funestus in Benin. Parasites \& Vectors, 1-12. https://doi.org/10.1186/s13071016-1723-y

18. El, A., Lalami, O., Fez, H. T., \& Saad, I. K. (2010). Pollution des eaux de surface de la ville de Fès au Maroc: Typologie, origine et conséquences.Larhyss journal n ${ }^{\circ} 09$; pp55-72.

19. Etang, J., Pennetier, C., Piameu, M., Bouraima, A., Chandre, F., Awono-ambene, P., Corbel, V. (2016). When intensity of deltamethrin resistance in Anopheles gambiae s . 1. leads to loss of Long Lasting Insecticidal Nets bio-efficacy: a case study in north Cameroon. Parasites \& Vectors, 1-10. https://doi.org/10.1186/s13071-016-1420$\mathrm{X}$

20. Fahala, A.A, 2006 Monographie de la commune de Zè; Programme d'appui au démarrage des communes, Afrique Conseil p8

21. Fanello, C., Petrarca, V., Della Torre, A., Santolamazza, F., Dolo, G., Coulibaly, M., Coluzzi, M. (2003). The pyrethroid knock-down resistance gene in the Anopheles gambiae complex in Mali and further indication of incipient speciation within An. gambiae s.s. Insect Molecular Biology, 12(3), 241-245. https://doi.org/10.1046/j.13652583.2003.0040

22. Fontenille, D., Cohuet, A., Awono-Ambene, P., Kengne, P., AntonioNkondjio, C., Wondji, C., \& Simard, F. (2005). Vecteurs de paludisme: du terrain à la génétique moléculaire Recherches en Afrique. Rev Epidemiol Sante Publique, 53, 283-290. 
23. Gillies, M., \& De Meillon, B. (1968). The Anophelinae of Africa south of the Sahara (Ethiopian zoogeographical region). The South African Institute for Medical Research, 2, 101-143.

24. Gnanguenon, V., Agossa, F. R., Badirou, K., Govoetchan, R., Anagonou, R., Oke-agbo, F., ... Akogbeto, M. C. (2015). Malaria vectors resistance to insecticides in Benin: current trends and mechanisms involved. 1-14. https://doi.org/10.1186/s13071-0150833-2

25. Gouagna, L. C., Dehecq, J., Girod, R., Boyer, S., \& Lempérière, G. (2011). Spatial and temporal distribution patterns of Anopheles arabiensis breeding sites in La Reunion Island - multi-year trend analysis of historical records from 1996-2009. Parasites \& Vectors, 4(1), 121. https://doi.org/10.1186/1756-3305-4-121

26. Hadji, M., Belghyti, D., Elomari, F., \& El, M. (2013). Étude de la dynamique stationnelle des populations des culicidés dans la province de Sidi Slimane ( Maroc ) Afrique Science: Revue Internationale des Sciences et Technologie 09(1), 128-139.

27. Hemingway, J., Hawkes, N. J., Mccarroll, L., \& Ranson, H. (2004). The molecular basis of insecticide resistance in mosquitoes. Insect Biochemistry and Molecular Biology, 34, 653-665. https://doi.org/10.1016/j.ibmb.2004.03.018

28. Koumba, A. A., Roland, C., Koumba, Z., Nguema, R. M., Djogbenou, L. S., Ondo, P. O., Iret, T. (2018). Distribution spatiale et saisonnière des gîtes larvaires des moustiques dans les espaces agricoles de la zone de Mouila, Gabon Int. J. Biol. Chem. Sci. 12(4): 1754-1769 ; http://www.ifgdg.org

29. Kudom, A. A. (2015). Larval ecology of Anopheles coluzzii in Cape Coast , Ghana: water quality, nature of habitat and implication for larval control. Malaria Journal, 1-13. https://doi.org/10.1186/s12936015-0989-4

30. Mbida, A. M., Awono-Ambene, P. N. A. P., Wopo, H. S., Talipouo, A., Tchoffo Fobasso, R., Dongmo, A., Mimpfoundi, R. (2018). Contribution à la gestion durable du bassin versant du Wouri par une étude de la répartition de sa faune culicidienne (Littoral-Cameroun). Revue d'Ecologie (Terre et Vie), 73(4), 550-558.

31. Mesari, N. (1997). Les peuplements culicidiens de la ville de Mohammedia et des régions avoisinantes: caractérisation hydrologique et hydrochimie des principaux gîtes dynamiques spatiotemporelle. cycle, Univ. Mohammed V, Fac. Sc

32. Mouchet, J., Carnevale, P., Coosemans, M., Jul-, J., Manguin, S., Richard-lenoble, D., ... Eurotext, L. (2004). Biodiversité du paludisme dans le monde: Paris Editions John Libbey Eurotext. 312. 
33. Nanagbé F, 2006 Monographie de la commune de Zè; Programme d'appui au démarrage des communes, Afrique Conseil P 9

34. Ossè, R., Aikpon, R., Padonou, G. G., Oussou, O., Yadouléton, A., \& Akogbéto, M. (2012). Evaluation of the efficacy of bendiocarb in indoor residual spraying against pyrethroid resistant malaria vectors in Benin: results of the third campaign. Parasites and vectors 1-10. https://doi.org/10.1186/1756-3305-5-163

35. Ouattara, A. F., Dagnogo, M., Constant, E. A. V, Koné, M., Raso, G., Tanner, M., ... Koudou, B. G. (2014). Transmission of malaria in relation to distribution and coverage of long-lasting insecticidal nets in central Côte d , Ivoire. Malaria journal 13:109 https://doi.org/10.1186/1475-2875-13-109

36. G. G. Padonou, M. Sezonlin, G. L. Gbedjissi, I. Ayi, R. Azondekon, A. Djenontin, S. Bio-Bangana, O. Oussou, A. Yadouleton, D. Boakye and M. Akogbeto. (2011). Biology of Anopheles gambiae and insecticide resistance: Entomological study for a large scale of indoor residual spraying in South East Benin. Journal of Parasitology and Vector Biology 3(4), pp. 59-68 https://doi.org/10.5897/JPVB11.018

37. Rodhain, F., \& Pérez, C. (1985). Précis d'entomologie médicale et vétérinaire, Notion d'épidémiologie des maladies à vecteurs. Maloine s.a.

38. Rousseau Djouaka, Innocent Djègbè, Romaric Akoton, Razack Adéoti, Genevieve. Tchigossou. (2018). Genes involved in multiple insecticide resistance in Anopheles gambiae and Anopheles coluzzii from Kpomé a tomatoes growing area in the southern Benin. Int J Biol Med Res 6(2), 6224-6231. www.biomedscidirect.com

39. Rowland, M., Boko, P., Odjo, A., Asidi, A., Akogbeto, M., \& Guessan, R. N. (2013). A New Long-Lasting Indoor Residual Formulation of the Organophosphate Insecticide Pirimiphos Methyl for Prolonged Control of Pyrethroid-Resistant Mosquitoes: An Experimental Hut Trial in Benin. Plos One 8(7), 1-10. https://doi.org/10.1371/journal.pone.0069516

40. Saliha, B., Wafa, H., Khellaf, R., \& Fatiha, M. (2018). Études entomologique et typologique des gîtes larvaires des moustiques (Diptera: Culicidae) dans la région de Bousaâda (Algérie). Bulletin de La Société Royale Des Sciences de Liège, 87, 112-120.

41. Silver, J. B., \& Service, M. W. (2007). Mosquito Ecology: Field sampling methods. Springer Eds. books.google.com

42. OMS. (2017). Procédures pour tester la résistance aux insecticides chez les moustiques vecteurs du paludisme Seconde édition. Programme mondial de lutte antipaludique, Genève.; http://www.who.int/malaria/fr 
43. OMS. (2018). Progrès vers l'élimination du paludisme: rapport du deuxième forum mondial des pays éliminant le paludisme. Genève. http://www.who.int/malaria/fr

44. OMS. (2019). World health statistics overview 2019: monitoring health for the SDGs, sustainable development goals. Geneva. http://www.who.int/malaria/fr 